\title{
A Case Report: Organophosphate Insecticide Intoxication in a Family
}

\author{
Aliasghar Manouchehri ${ }^{1^{*}}$, Reza Mohseni ${ }^{2}$, Tahereh Hejazian ${ }^{2}$ \\ 1. Department of Internal Medicine, School of Medicine, Babol University of Medical Sciences, Babol, Iran. \\ 2. Student Research Committee, Babol University of Medical Sciences, Babol, Iran.
}

Article info:

Received: 11 Jan. 2017

Accepted: 25 Apr. 2017

\section{Keywords:}

Organophosphate, Intoxication, Pediculosis capitis
Citation: Manouchehri A, Mohseni R, Hejazian T. Organophosphate Insecticide Intoxication in a Family; A Case Report. International Journal of Medical Toxicology \& Forensic Medicine. 2017; 7(3):185-188. https://doi.org/10.18869/nirp.ijmtfm.7.3.185

: https://doi.org/10.18869/nirp.ijmtfm.7.3.185

\section{Introduction}

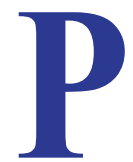

ediculosis capitis (head lice) is a global problem and contaminates all age groups, especially children [1]. The prevalence of infection with head lice has been $6 \%$ to $30 \%$ according to reports from different regions [2]. In this disease, blood of the infected person is sucked by lice several times a day, while insects' saliva is repeatedly injected into the victim's body. Therefore, its toxic effects on infected persons may cause fatigue, irritation, and feelings of laziness [3].
Drugs used to treat lice, contain permethrin. Sometimes shampoos containing lindane or malathion are used to treat resistant lice that must be prescribed by a physician [4]. The mortality rate due to organophosphorate poisoning have been reported between $3 \%$ and $25 \%$. The rate in Asia is estimated to be 5\% to 30\% [5]. More Organophosphate (OP) insecticides poisoning occur by ingestion, skin contact, inhalation, and rarely through intramuscular or intravenous routes. OP compounds include chemicals that inhibit the enzyme acetylcholinesterase that leads to the accumulation of acetylcholine in the cholinergic synapses [6]. Herein, we report a couple of siblings and their mother who, upon topical exposure with an organo-

* Corresponding Author:

Aliasghar Manouchehri, PhD

Address: Department of Internal Medicine, School of Medicine, Babol University of Medical Sciences, Babol, Iran.

Tel: +98 (911) 3128920

E-mail: drmanouchehri@yahoo.com 
phosphate solution, developed severe OI associated with Central Nervous System (CNS) suppression.

\section{Case Report}

Case 1

After topical exposure of toxic insecticide (organophosphate diazinon) in the bathroom which was used for head lice infestation, a 31-year-old female patient from rural area of Babol City was referred to tertiary hospital with weakness, lethargy, and vomiting. The patient's vital signs at admission were as follows: temperature: $36.8^{\circ} \mathrm{C}$; HR: 80 beats per minute; respiratory rate: 22 breaths per minute; and BP: 120/80 mm Hg and her physical examination was normal. The results of clinical tests were as follows: BUN: $16 \mathrm{mg} / \mathrm{dL}$; Cr: $0.9 \mathrm{mg}$ / dL; WBC count: 6400; hemoglobin: $13.4 \mathrm{~g} / \mathrm{dL}$; platelets: 254000; magnesium: 2.6; phosphorus: 2.6; CPK: 130; LDH: 360 and; calcium: 10. Remedial management were taken including administration of atropine and ondansetron. The patient was discharged in good condition.

\section{Case 2}

A 10-year-old boy (son of case 1) was admitted because of skin contact with the pesticide malathion prescribed because of his head lice. On admission, the patient had loss of consciousness so CPR was immediately begun and the patient was connected to the ventilator. Patient's vital signs were as follows: temperature: $37.5^{\circ} \mathrm{C}$; HR: 100 beats per minute; $\mathrm{BP}: 95 / 45 \mathrm{~mm} \mathrm{Hg}$; and $\mathrm{PO}_{2}$ : 98\%. On physical examination, GCS score of patient was about 7 with miotic pupils. There was generalized crackles in all fields of lung in auscultation. In heart auscultation there was tachycardia. Abdominal examinations were normal. Initial managements included washing of head, face, and body, suction of secretions, with shaving head. Aggressive serum therapy with prescribed antidote (atropine and pralidoxime) was started and the patient was transferred to the ICU. The patient had seizures twice which were controlled by diazepam. The results of laboratory tests for patient were as follows: WBC count: 6600; Hb: $11.7 \mathrm{~g} / \mathrm{dL}$; platelets: 266000; BUN: $10 \mathrm{mg}$ / dL; Cr: 0.6 mg/dL; Bs: 104; Na: 136; K: 3.5; PH: 7.43; $\mathrm{PCO}_{2}: 40$; and $\mathrm{HCO}_{3}: 26.6$. In progress, the state of patient's condition was improved and discharged.

\section{Case 3}

A 7-year-old girl (daughter of case 1) was brought to the emergency room at the same time with the second case with loss of consciousness and cardiorespiratory ar- rest. Her mother washed her hair with spraying insecticides (OPs are probably diazinon groups) to remove her head lice infestation.

She was intubated immediately and after resuscitation for about 15 minutes, the patient's response to treatment appeared and her cardiac electrical activity restart. Results of clinical examination of the patient were as follows: temperature: $37.5^{\circ} \mathrm{C}$; HR: 124 beats per minute; BP, 85/90 mm Hg; and $\mathrm{SPO}_{2}$ : 97\%. Also she had GCS score of 5, mydriatic pupils unresponsive to light, generalized crackles in lung auscultation, tachycardia in heart auscultation, and no abnormality in abdominal examination. Initial managements included washing her head, face and body, shaving her head, and suction of secretions. Serum therapy was begun aggressively and treatment with prescribed antidotes (atropine and pralidoxime) were administered. Unfortunately, the girl was declared brain-dead despite our vigorous efforts.

\section{Discussion}

The most effective ways to treat head lice are using drugs like lindane, permethrin $1 \%$, crotamiton $10 \%$, malathion $0.5 \%$, sulfur cream $6 \%-12 \%$, as well as public education and health promotion in contaminated communities [7]. According to WHO classification, malathion and diazinon are in class 2 and class 3 respectively, so they should be used with great caution for head lice [8].

The most common cause of death in this kind of poisoning are respiratory failure due to respiratory centers depression, respiratory muscle weakness, suppression of the central nervous system, and bronchorrhea [10]. Diagnosis of OP is based on patient's history, clinical status, and laboratory tests. Clinical manifestation depends on the amount and route of exposure [5]. Signs and symptoms of acute poisoning with organophosphates start within 1-2 hours after the contact. However in some cases, especially in skin contact or poisoning with toxic agents with metabolically active form, clinical manifestations may be delayed. In this case, a person with mild clinical symptoms may become suddenly ill [11].

Clinical manifestations are divided into three categories: muscarinic, nicotinic symptoms, and central nervous system effects. Chemical pneumonia may occur as a result of exposure to these compounds and pulmonary aspiration. Muscarinic symptoms and signs include nausea, diarrhea, abdominal cramps, bronchospasm, meiosis, bradycardia, increased salivation, and severe sweating. Profuse sweating leads to dehydration and hypovolemic shock. Nicotinic symptoms include muscular 
fasciculation and tremors with weakness. Death may occur due to respiratory muscle paralysis. Due to nicotinic or muscarinic effects, blood pressure and heart rate could increase or decrease respectively. Central nervous system symptoms such as restlessness, seizures, and coma can occur, too [12]. Unlike adults, infants mainly present with acute CNS depression [6] and do not demonstrate the typical muscarinic effects. Symptoms such as fasciculation, bradycardia, and acute respiratory failure are more common in children [13].

Poisoning management with cholinesterase inhibitors include care and support, decontamination, improving oxygenation, removing the muscarinic signs and symptoms and prescribing the antidote and pralidoxime for regeneration of acetyl cholinesterase. One of the most important management procedures is reducing poisons absorption through skin and mucosa. Thus, during and after the patient's stabilization, decontamination of skin needs to be done. So that all contaminated clothing should be removed and all areas of exposed skin rinsed with cold water and soap [14]. Specific treatments include anti-muscarinic agents such as atropine and reactivation of cholinesterase by pralidoxime [15]. According to the previous studies on organophosphate effects, they can be very dangerous due to their easy accessibility and disregarding their safety handling by people. Therefore, simultaneous examination and treatment of people infected with lice in the household and emphasis of health care staff on patients' training and follow-up, can be helpful in treating this disease.

\section{Conclusion}

The striking similarity and timely fashion of the clinical presentation of these siblings and their mother suggested wrong beliefs among rural citizens about management of pediculosis capitis. Both children had some elements of CNS depression, respiratory difficulty, excessive sweating, and myosis of pupils. This constellation of findings is highly suggestive of a cholinergic toxidrome, and additional inquiry revealed exposure to organophosphate compounds. This report emphasizes that misuse of organophosphate compounds, even in cases of domestic application, may be life threatening. This can cause acute OI even through the skin contact. Management of OI consists of opening the airway; administration of oxygen, fluid, and atropine in increasing doses as well as pralidoxime; and decontamination of the patient's skin. The health care personnel should be aware of the early management and decontamination when taking care of contaminated patients.

\section{Acknowledgements}

This research did not receive any specific grant from funding agencies in the public, commercial, or not-forprofit sectors. The authors gratefully acknowledge the patients who allowed us to prepare this case report. We would also like to thank the Clinical Research Development Unit of Rouhani Hospital of Babol.

\section{Conflict of Interest}

The authors declared no conflicts of interest.

\section{References}

[1] Durand R, Bouvresse S, Berdjane Z, Izri A, Chosidow O, Clark JM. Insecticide resistance in head lice: Clinical, parasitological and genetic aspects. Clinical Microbiology and Infection. 2012; 18(4):338-44. doi: 10.1111/j.1469-0691.2012.03806.x

[2] Noroozi M, Saghafipour A, Akbari A, Khajat P, Khadem Maboodi AA. [The prevalence of pediculosis capitis and its associated risk factors in primary schools of girls in rural district (Persian)]. Journal of Shahrekord University of Medical Sciences. 2013; 15(2):43-52.

[3] Khopkar U, Madke B. Pediculosis capitis: An update. Indian Journal of Dermatology, Venereology, and Leprology 2012; 78(4):429. doi: 10.4103/0378-6323.98072

[4] Verma P, Namdeo C. Treatment of pediculosis capitis. Indian Journal of Dermatology. 2015; 60(3):238-47. doi: 10.4103/0019-5154.156339

[5] Thundiyil JG, Stober J, Besbelli N, Pronczuk J. Acute pesticide poisoning: a proposed classification tool. Bulletin of the World Health Organization. 2008; 86(3):205-9. doi: 10.2471/ blt.08.041814

[6] Blain PG. Organophosphorus poisoning (acute). BMJ Clinical Evidence; 2011: 2102. PMCID: PMC3217786

[7] Agency for Healthcare Research and Quality. Guidelines for the diagnosis and treatment of pediculosis capitis (head lice) in children and adults. Rockville: Agency for Healthcare Research and Quality; 2013.

[8] World health Organization. The WHO recommended classification of pesticides by hazard and guidelines to classification 2009. Geneva: World Health Organization; 2010.

[9] Moghadamnia A, Abdollahi M. An epidemiological study of poisoning in northern Islamic Republic of Iran. Eastern Mediterranean Health Journal. 2002; 8(1):88-94. PMID 15330564

[10] Nambiar MP, Gordon RK, Rezk PE, Katos AM, Wajda NA Moran TS, et al. Medical countermeasure against respiratory toxicity and acute lung injury following inhalation exposure to chemical warfare nerve agent VX. Toxicology and Applied Pharmacology. 2007; 219(2-3):142-50. doi: 10.1016/j taap.2006.11.002

[11] Reji KK, Mathew V, Zachariah A, Patil AKB, Hansdak SG Ralph R, et al. Extrapyramidal effects of acute organophos- 
phate poisoning. Clinical Toxicology. 2016; 54(3):259-65. doi: 10.3109/15563650.2015.1126841

[12] Aygun D, Onar M, Altintop B. The clinical and electrophysiological features of a delayed polyneuropathy developing subsequently after acute organophosphate poisoning and it's correlation with the serum acetylcholinesterase. Electromyography and clinical neurophysiology. 2002; 43(7):421-7. PubMed: 14626722

[13] El-Naggar AER, Abdalla MS, El-Sebaey AS, Badawy SM. Clinical findings and cholinesterase levels in children of organophosphates and carbamates poisoning. European Journal of Pediatrics. 2009; 168(8):951-6. doi: 10.1007/ s00431-0080866-z

[14] Yu Z, Sun B, Jian X. [Advances in diagnosis and treatment of intermediate syndrome of acute organophosphate poisoning (Chinese)]. Chinese Journal of Industrial Hygiene and Occupational Diseases. 2015; 33(3):235-7.

[15] ddleston M, Chowdhury FR. Pharmacological treatment of organophosphorus insecticide poisoning: the old and the (possible) new. British Journal of Clinical Pharmacology. 2015; 81(3):462-70. doi: 10.1111/bcp.12784 